\title{
Viscous stress distribution over a wavy gas-liquid interface
}

\author{
Matthieu A. André*, Philippe M. Bardet \\ Mechanical and Aerospace Engineering \\ The George Washington University \\ Washington, DC 20052, USA
}

\begin{abstract}
Viscous stress contributes to momentum transfer between two phases, which plays an important role in both industrial applications and environmental processes. Near a wavy interface, the flow is modulated and produces a spatially non-uniform normal and tangential viscous stress. This study presents measurements of these stresses at a liquid-gas interface populated with twodimensional millimeter scale waves performed with multiphase particle image velocimetry. Large datasets enable conditional phase-averaging of the data based on wave steepness, which increases the precision of the results and allows statistical analysis. For the first time at this scale, the spatial distribution of normal and tangential viscous stress is obtained for a large range of wave steepness ( $a k=0$ to 1 , with $a$ the amplitude and $k$ the wavenumber). As the steepness increases, the mean shear stress over a wavelength decreases in magnitude, while the normal viscous stress increases. These trends are linear for $a k<0.6$, and correlations are proposed. At $a k>0.7$, flow separation is observed in the gas phase near the troughs and drastically alters the viscous stress distribution.
\end{abstract}

Keywords: two phase flow, shear stress, viscous stress, PIV, wavy flow

\section{Introduction}

In many industrial applications such as nuclear reactors safety, oil/gas pipelines, ship hydrodynamics, and cavitating flows, it is necessary to estimate momentum transfer between two phases, typically a gas and a liquid (Fabre et al., 1987; Whalley, 1996; Kawaji, 1998; Ishii and Hibiki, 2010). Winddriven waves are an important topic in environmental flows (Banner, 1990; Peirson, 1997; Veron et al., 2007; Siddiqui and Loewen, 2007; Birvalski et al., 2015). The transfer can be either gas- or liquid-driven, through pressure (form drag) and viscous (normal and tangential) stresses. The relative contributions depend on the interface profile and flow field around it. At a curved, unsteady, uncontaminated two dimensional (2D) interface, the tangential and normal stresses can be written in 2D curvilinear coordinates according to Eq. 1 and Eq. 2, respectively (Schlichting and Gersten, 2000). $s$ and $n$ refer to the tangential and normal components in the curvilinear coordinates system defined in Fig. 1. $\kappa$ and $u$ are the surface curvature and velocity, respectively, and $\mu$ the dynamic viscosity of the phase of interest. $p$ is the pressure on the gas side of the interface.

$$
\begin{gathered}
\tau=\mu\left(\left.\frac{\partial u_{s}}{\partial n}\right|_{n=0}-\kappa u_{s}+\frac{\partial u_{n}}{\partial s}\right) \\
\sigma=-p+\left.2 \mu \frac{\partial u_{n}}{\partial n}\right|_{n=0}=-p+\sigma_{v}
\end{gathered}
$$

\footnotetext{
${ }^{*}$ Corresponding author

Email address: matandre@gwu.edu (Matthieu A. André)
}

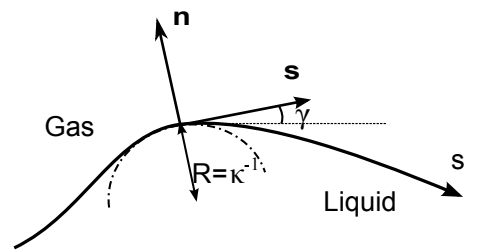

Figure 1: Curvilinear coordinates system used in the present study. $\gamma$ is the surface angle with respect to the horizontal

In addition to the surface normal gradient of tangential velocity (first term of right hand side), Eq. 1 shows that curvature and surface tangent velocity gradient also contribute to the shear stress. These last two terms are actually equal to half the surface vorticity in the liquid for a free surface (Lundgren and Koumoutsakos, 1999). They are usually neglected at macroscale (Veron et al., 2007; Peirson, 1997), but can significant contribute to the overall shear stress at capillary scale. The normal stress, Eq. 2, includes the contribution of pressure, as well as a viscous term, labeled $\sigma_{v}$. For a rising bubble, the later can be the dominant term for momentum transfer (Moore, 1959).

Previous experimental works have mostly focused on obtaining empirical correlations to predict global relevant quantities (drag, transfer of momentum, heat, and mass, pressure drop), based on general flow features such as mean velocity, mean pressure gradient, mean surface roughness or level of turbulence (Lopez (1994); Taitel and Dukler (1976); Belcher and Hunt (1993); Ayati et al. (2014)). These engineering models are practical, but can be inaccurate because not all the physical flow features are modeled; thus the validity domain and appli-

May 21, 2016 
cability of such correlations are limited. The readers can refer to Decarre et al. (2008) and Line and Lopez (1997) for examples of models for the interfacial shear. Detailed experimental data are also required for validating interface tracking methods in high-fidelity CFD codes.

In this paper, momentum transfer through viscous stress at a clean interface populated with 2D capillary scale disturbances is investigated using an experimental technique previously developed based on particle image velocimetry (PIV) coupled with planar laser induced fluorescence (PLIF) profilometry. In order to obtain statistically significant results for a wide range of steepness, data are extracted from two flow regimes close in $R e$, but showing very different steepness distributions, and then conditionally averaged based on wave steepness. Results for the spatial distribution of normal and tangential viscous stresses along the surface of capillary scale waves are presented. Empirical correlations for the mean stresses versus steepness over a wavelength are obtained, and effect of flow separation in the trough at high wave steepness is discussed.

\section{Experiment}

\subsection{Facility}

The experiment is a recirculating water channel. The flow is conditioned to minimize turbulence and non-uniformity, and is accelerated through a two-dimensional contoured nozzle to generate a top-hat rectangular jet with high velocity uniformity and low level of initial disturbances $(<0.6 \%$ of $U)$. The jet at the nozzle exit is $20.3 \mathrm{~mm}$-thick by $146 \mathrm{~mm}$-wide with laminar boundary layers, and discharges into an open-top transparent channel surrounded by quiescent air, which is subsequently entrained through momentum transfer, as depicted in Fig. 2.

Measurements are performed at a downstream location between $x=19$ and $29 \mathrm{~mm}$, and at a spanwise distance $y=40$ $\mathrm{mm}$ from the channel side wall. The free stream velocity, $U$, can be controlled from 1 to $10 \mathrm{~m} / \mathrm{s}$, and is set at $U=2.72$ and $3.00 \mathrm{~m} / \mathrm{s}$ in the present study, resulting in a momentum thickness at the nozzle exit, $\theta$ of $0.056 \mathrm{~mm}$ and $0.053 \mathrm{~mm}$, and $R e_{\theta}=U \theta / v=151$ and 159 respectively.

Boundary layer thickness along the nozzle walls is predicted by a potential flow solver coupled with a boundary layer momentum integral calculation. Results were validated with PIV measurements at the nozzle exit and agree within 5\%. More details of the facility and characterization of the initial velocity profiles are presented in André (2014).

The boundary layers at the channel bottom and side walls have a thickness below $0.6 \mathrm{~mm}$ in the measurement region, which is 34 times and 67 times smaller than the channel depth and distance to the side walls respectively. For instance in Fig. 3 (b), the flow is considered uniform below $z / \theta=-15$, while the bottom wall is located at $z / \theta=-344$. Therefore bottom and side walls have a negligible effect on the flow in the present measurement region.

The boundary layer generated at the upper wall of the nozzle becomes a surface shear layer upon exiting the nozzle, Fig. 3 (a), as the boundary condition changes from no-slip at the nozzle wall to shear-free at the free-surface. Fig. $3(b)$ shows a

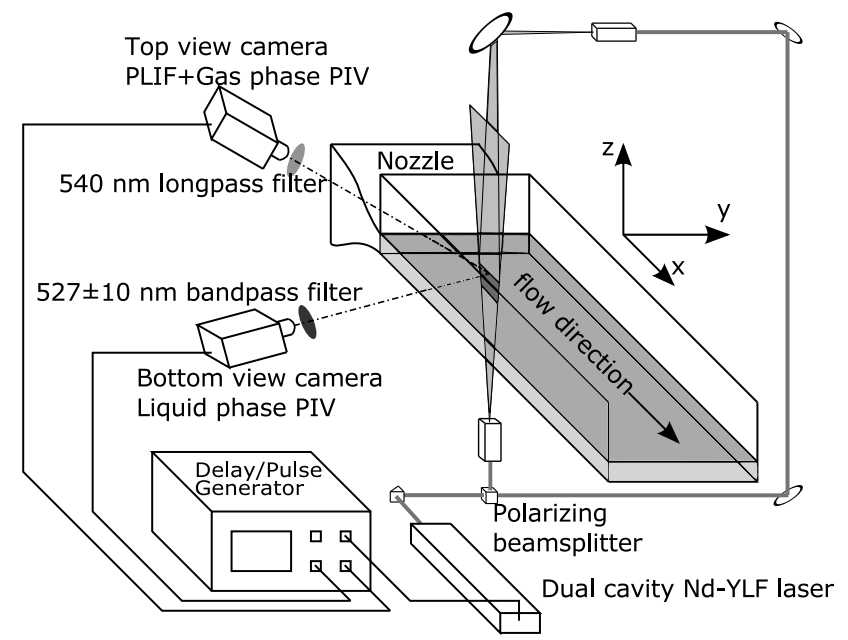

Figure 2: Schematic of the experimental setup

measurement of such shear layer in the case of a flat surface. Relaxation of the velocity profile due to the change of boundary condition is visible as the flow moves downstream, but remains small $(<5 \% U)$ over the field of view (FOV).

Such shear layer relaxation can become unstable (Brennen, 1970) for $R e_{\theta}>140$ in the present experiment, and the subsequent roll-up into vortices forces waves at the surface (André and Bardet, 2015a). The 2D linear theory analysis of Brennen (1970) states that the most unstable wavelength scales with $\theta$. More precisely, the dimensionless frequency $\bar{f}=2 \pi f / U$ tends towards $\bar{f}_{\text {inviscid }}=0.175$ in the inviscid limit. These waves travel downstream at the same speed as the vortices, which is about $0.8 U$ in the present data.

The $2 \mathrm{D}$ nature of the surface waves is assessed using topdown view flash photographs as shown in Fig. 4. In the measurement region, the waves show slight spanwise fluctuations at a wavelength of 2 to 3 times the streamwise wavelength, and shifted by up to 10 to $20 \%$ of the streamwise wavelength. This is deemed acceptable to consider the present flow as 2D in the measurement region. $R e_{\theta}$ higher than 170 leads to a surface quickly transitioning to $3 \mathrm{D}$, which could not be fully investigated with the present 2D diagnostics.

\subsection{Instrumentation and data processing}

The near-surface flow is investigated using a combination of dual-phase time-resolved PIV and PLIF profilometry presented in André and Bardet (2015b), and schematically shown in Fig. 2. Each phase is instrumented with a dedicated camera (Phantom V311 and 710) and laser (Photonics DM30-527), which overlap in the region of interest. The FOV is $10 \mathrm{~mm}$ wide, which corresponds to about 5 wavelengths. The profilometry is based on PLIF, where a fluorescent dye is homogeneously dissolved in the liquid. Masks are generated from these surface profiles to process the PIV images, which are recorded with a $1.6 \mu$ s delay between gas and liquid phases to avoid cross-talk of the signals. PIV tracers are $2 \mu \mathrm{m}$ silver coated glass spheres in the liquid phase, and $0.5 \mu \mathrm{m}$ Kiton reddoped polystyrene latex spheres in the gas phase. 
(a)

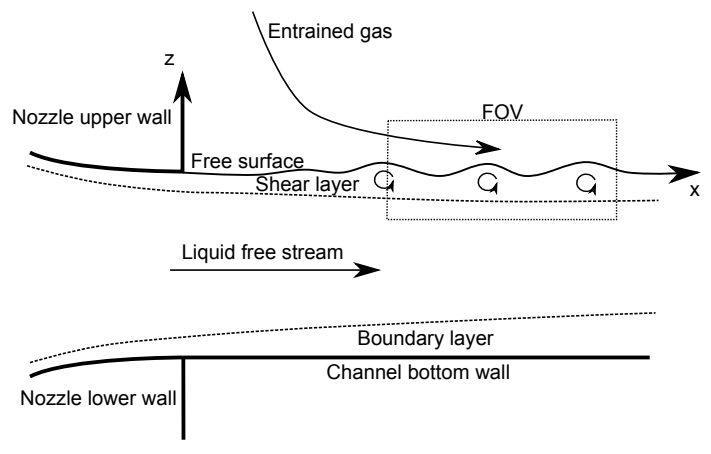

(b)

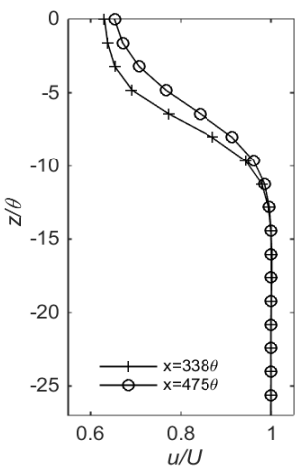

Figure 3: $(a)$ : 2D cross section schematic of the flow near the nozzle exit. FOV: field of view. Thickness of boundary layers in the nozzle and shear layer below the surface are exaggerated for clarity. $(b)$ : Measured mean liquid velocity profiles below the surface at the boundaries of the FOV for a flat surface regime $(U=2.42$ $\mathrm{m} / \mathrm{s}, \theta=0.059 \mathrm{~mm})$
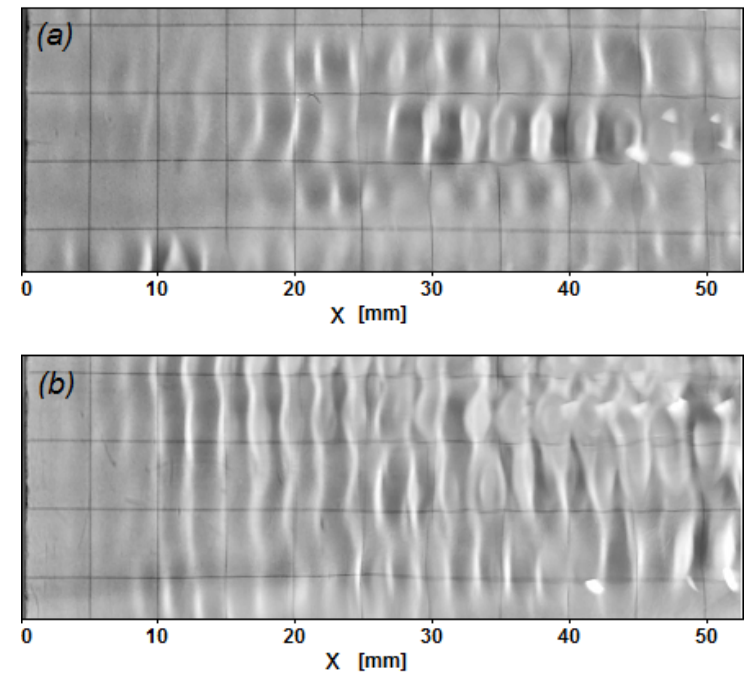

Figure 4: Top view photographs of the flow at the nozzle exit (located at $x=0$ $\mathrm{mm}$ ) for $R e_{\theta}=151(a)$ and $R e_{\theta}=159(b)$. Measurement region is between $x=19$ and $29 \mathrm{~mm}$, where the flow is still considered 2D

High magnification optics and high speed imaging allow resolving the velocity down to a spatial and temporal resolution of $100 \mu \mathrm{m}$ and $100 \mu \mathrm{s}$, respectively using adaptive interrogation windows $32 \times 32$ pixels in size, and overlapping by $75 \%$.

At the interface, velocity and vorticity from the gas and liquid sides can be interpolated knowing the surface profile. Surface curvature, necessary for the shear stress calculation Eq. 1, is computed with an adaptive curve fitting algorithm described in André and Bardet (2014). Uncertainty was assessed with synthetic images, and showed to be below $12 \%$ for $1 /(|\kappa| \Delta x)>6$, with $\Delta x=11 \mu \mathrm{m}$ the PLIF spatial resolution. This corresponds here to $|\kappa|<15(\mathrm{~mm})^{-1}$, or $|\kappa| \lambda<30$, which is larger than in the present results $(|\kappa| \lambda<15)$.

Shear stress results presented in this work are obtained using only gas phase data according to Eq. 1. This scheme offers relatively good performances because stress is easier to measure in the gas as a result of the thicker Stokes viscous layer. The latter stems from locally oscillating flow induced by sur-

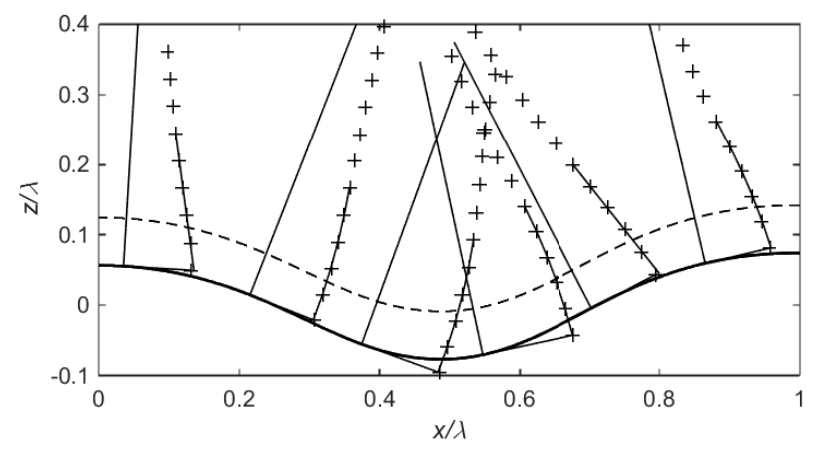

Figure 5: Details of the curve fit of tangential velocity along surface normal direction in the case of a wavy surface. Dash line indicates the Stokes layer

face waves (Longuet-Higgins, 1992), and scales with the square root of the kinematic viscosity. Its thickness is around $0.16 \mathrm{~mm}$ in the present flow, and is shown in Fig. 5. Surface normal and tangential velocities are extrapolated at the surface from the average of the neighboring PIV data-points. Calculation of the surface normal gradients (first term of Eq. 1, and second term of Eq. 2) require interpolation of the velocity field on a surface normal line. In the case of a flat or low steepness wave, the 5 points closest the surface are fitted with a linear regression. If the fit is a good match $\left(R^{2}>0.95\right)$, the surface normal gradient is simply the line slope.

At larger steepness, the surface induces a secondary flow that modulates the velocity field within the Stokes layer, and requires a second order polynomial fit of the velocity profile to resolve the gradient at the surface, as illustrated in Fig. 5.

The computation of the shear stress results in noisy data, in part due to noise amplification by the spatial derivations required for the velocity gradients and surface curvature (Foucaut and Stanislas, 2002). Uncertainties as large as $25 \%$ are reported for single shot measurements, and motivate the need to average data to reduce random error (precision error). 


\subsection{Data binning and conditional averaging}

By averaging, data noise is reduced and meaningful statistics about the flow are gained. Phase averaging is commonly used for periodic flows to identify main flow structures and has also been used for classifying surface waves, as done by Siddiqui and Loewen (2010) and Birvalski et al. (2014).

In the present case, the flow is repeatable and can be approximated as 2D, as shown in Fig. 4, but there is spread in the data due to sensitivity of the instability to initial disturbances. Thus, wave profiles differ slightly from wave to wave at a given location. Since this study focuses on data at and near the surface, a consistent way to extract and sort the data is defined to perform conditional averaging: data are phase-averaged over a wavelength with a condition on wave steepness, as described below.

The waves are first extracted between adjacent crests (defined where the surface slope is zero). It is shown in the results section that flow separation can occur in the gas phase near the trough, and affects the shear stress there. This justifies the choice of this investigation domain versus a trough-to-trough approach.

The distribution of wavelength, $\lambda$, is compared to the theory of Brennen (1970). Viscous effects tend to slightly decrease the frequency and the peak of the distribution of measured frequency is at $\bar{f} / \bar{f}_{\text {inviscid }}=0.89$ and 0.97 (or a wavelength of 2.25 $\mathrm{mm}$ and $1.96 \mathrm{~mm}$ ) for $R e_{\theta}=151$ and 159 , respectively. More than $95 \%$ of the detected waves have a wavelength of $\pm 50 \%$ of the predicted inviscid limit frequency, and about $80 \%$ are within $\pm 30 \%$ of the most represented wavelength. Only waves matching the latter criterion are selected for further processing. Using Brennen's relation, the flow regimes can also be expressed in term of Reynolds number based on disturbances wavelength: $R e_{\lambda}=\left(2 \pi / \bar{f}_{\text {inviscid }}\right) R e_{\theta}=5.42 \times 10^{3}$ and $5.71 \times 10^{3}$ for $R e_{\theta}=151$ and 159 , respectively.

The main parameter differentiating the waves is the steepness, $a k=2 \pi a / \lambda$ with $a=\left(c_{1}+c_{2}-2 t\right) / 4$ the mean half crest-totrough amplitude, see Fig. 6 for definition of parameters. This formulation accounts for the asymmetry between upstream and downstream facing slope of the wave profile resulting from the vortex motion below the surface (André and Bardet, 2014). The equivalent Reynolds number based on disturbances amplitude is $R e_{a}=(a k / 2 \pi) R e_{\lambda}=\left(a k / \bar{f}_{\text {inviscid }}\right) R e_{\theta}$. The waves are sorted in bins defined by a range of steepness. Several bins size are tested, and the value of 0.1 is selected. This bin size is used to plot the steepness distributions for $R e_{\theta}=151$ and $R e_{\theta}=159$ in Fig. 7. Note that the distribution shape is sensitive to the bin size, and that choosing a smaller bin size would reveal a log-normal distribution for the $R e_{\theta}=151$ regime as shown in André and Bardet (2015b) for a bin size of 0.06. For the current purpose of studying the influence of steepness on shear stress, a bin size of 0.1 is more appropriate and increases the number of samples per bin.

The number of waves in each bin can be directly read from these histograms. Overall, the datasets include 3692 waves for $R e_{\theta}=151$ and 6253 waves for $R e_{\theta}=159$. Most waves are below $a k=0.3$ for the first case, and between $a k=0.1$ and 0.7

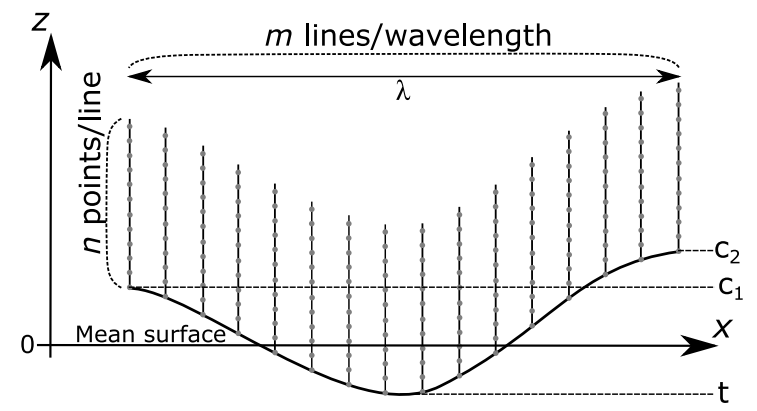

Figure 6: Grid for interpolation of the PIV data in the gas phase. $c_{1}$ and $c_{2}$ are the upstream and downstream crest elevations, respectively, and $t$ is the trough elevation. Sampling scheme uses $m=n=50$ in this study

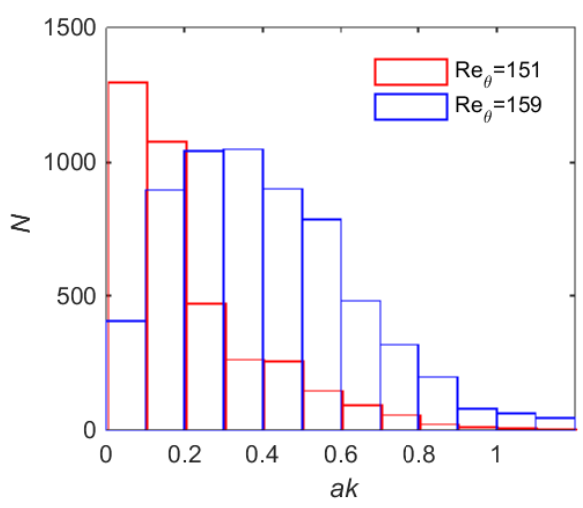

Figure 7: Distribution of wave steepness for $R e_{\theta}=151$ (red) and $R e_{\theta}=159$ (blue). $N$ is the number of samples in each bin. Bin size is 0.1

in the second case. Despite the small variation in $R e_{\theta}$, the two regimes exhibit a very different distribution of wave steepness because of the exponential nature of unstable growth. Then, as the surface accelerates, the growth rate drops until the flow reaches neutral stability (Brennen, 1970). This last part of the growth is observed in the present FOV. The waves then reach neutral stability and are convected downstream, as visible in Fig. 4. This large variation in steepness is beneficial for investigating a larger range of steepness than would be possible with only one case, providing the two regimes have similar normalized boundary conditions (surface profile and velocity). This is checked in the first part of the results, Sec. 3.1.

Waves within a bin have slightly different wavelengths, steepnesses and profiles, therefore a procedure is defined to consistently sample and compute the phase averaged data on identical grids. Each wave is horizontally discretized in $m=$ 50 uniformly distributed points, and the surface data (profile, curvature, velocity, and shear stress) are interpolated at these points. Velocity field data are interpolated along vertical lines aligned with the surface points previously defined. Field data are vertically interpolated on $n=50$ points with similar spacing as for the profile. This grid is illustrated in Fig. 6 for the gas phase. A similar interpolation is performed in the liquid phase. The number of data-points is enough to avoid undersampling the PIV data (about 20 vectors/wavelength). For each bin, surface and field data are averaged based on these grids. 
Root mean square of the difference to the mean (simply noted RMS) is also computed for each quantity. Averaged result is reconstructed on the mean profile for each steepness bin (phase average of the surface profiles).

\section{Results}

Phase averaged results are presented here. Bins must contain sufficient number of independent samples to obtain meaningful statistics. Data taken in time series, as done here, are correlated. However, as a wave travels over the FOV, it is sorted in different bins as a consequence of its growth. It is estimated that the steepness of a wave increases by less 0.1 (bin width) in about $1 \mathrm{~ms}$, which corresponds to 10 PIV samples. Therefore, the number of independent samples in a bin is at least a tenth of the total number of waves in that bin. The minimum number of independent samples a bin must contain is arbitrary set to 16 to give a ratio of at least $\sqrt{16}$ between samples standard deviation and standard error on the calculated mean according to the central limit theorem. Bins containing more than 160 PIV samples include bins up to $a k=0.6$ and $a k=0.9$ for $\operatorname{Re}_{\theta}=151$ and $R e_{\theta}=159$, respectively.

Lengths, velocities, and stresses are normalized by $\lambda, U$, and $\rho U^{2} / 2$, respectively. In the following, bins are referred to their mid-value, for instance the bin $0.1 \leq a k<0.2$ is labeled $a k=$ 0.15 .

\subsection{Surface profile and velocity}

Conditional phase-averaged surface profile, curvature, and velocity are shown as solid lines for three ranges of steepness (bins $a k=0.15,0.45$, and 0.75) in Fig. 8 for $R e_{\theta}=151$ (red) and $\operatorname{Re}_{\theta}=159$ (blue). For all steepnesses the surface profiles are very similar, while the RMS of the deviation to the mean, showed as dashed lines, increase with steepness.

Curvature plots are also consistent between the two regimes for $a k=0.15$ and 0.45. Large deviations are visible in the trough for $R e_{\theta}=151$ in the $a k=0.75 \mathrm{bin}$. This is a consequence of both the small number of samples (56) and sensitivity of curvature calculation on surface profile, and justifies the choice to not rely on bins with low number of samples.

The trend in the surface normal velocity is very consistent over the bins, with an increasing of the modulation of $u_{n}$ as $a k$ increases. In the low amplitude case (bin $a k=0.15$ ), surface profile and velocities are very close to sine and cosine functions, respectively, and the flow can still be described using linear approximation. For the bin $a k=0.45$, the curvature plot reveals that the trough is actually sharper than the crest, indicating surface profile is departing from cosine function. This feature is characteristic of capillary waves (Crapper, 1957), and becomes more pronounced as the wave steepens. Departure from the sine-like function is also visible for the surface velocity. Surface tangent velocity presents slightly more discrepancy, with the case $\operatorname{Re}_{\theta}=151$ being lower by up to $10 \%$ than $R e_{\theta}=159$ towards the downstream half of the wave. The difference is still within the standard deviations. Söderberg and Alfredsson (1998) studied relaxation of a flat laminar jet, and showed that normalized surface velocity is self similar when the downstream distance is scaled by $R e$ for $100<R e<10,000$ the Reynolds number based on boundary layer thickness. For the present cases, that would translate into a difference of $5 \%$ in surface velocity at the same location if the surface were flat. Nevertheless, the overall agreement in surface profile and velocity between the two regimes is satisfactory, and allows the results to be combined in order to present shear stress results that span a larger range of steepness.

Velocity vector fields are averaged following the method described in Sec. 2.3 for both phases, and plotted with respect to the mean surface profile. Examples of such vector fields for $R e_{\theta}=159$ and bins $a k=0.15,0.45$, and 0.75 are shown in Fig. 9. Velocity is displayed in the frame of reference moving with the wave in which the surface profile is close to a flow streamline. The average phase speed is measured by tracking the trough location of a wave over several frames. Over the FOV, the wave phase speed is constant within 5\%. The nonCartesian sampling grid defined in Fig. 6 is clearly visible and can be misleading. For instance, vectors away from the interface seem to form a wavy pattern; this pattern, however, is due to the grid, and the vectors point mainly horizontally in these regions. Swirling strength -useful for identifying vortices (Zhou et al., 1999)- is computed, normalized by $U^{2} / \lambda^{2}$, and displayed as a contour plot in each phase. Primary vortex resulting from the liquid shear layer roll-up is visible below the crest and its strength is similar for all steepnesses. A vortical motion is also visible attached to the top of the crest in the gas phase. Its swirling strength magnitude is coincidentally similar to that of the liquid for $a k=0.15$. At higher wave steepness, the gas phase swirling strength becomes stronger by a factor of about 2 , likely due to the larger amount of momentum transferred during the wave growth.

\subsection{Spatial distribution of viscous stress}

The interfacial viscous shear and normal stresses are calculated for each wave according to the method described in Sec. 2.2. Once the waves are sorted into appropriate bins, viscous stresses are averaged and normalized according to Sec. 2.3 and presented in Fig. 10 and Fig. 11 for the normal and tangential components, respectively. The spatial distribution of stress along a wave is plotted in blue for $R e_{\theta}=159$ for $a k$ bins ranging from 0.05 to 0.85 , and in red for $\operatorname{Re}_{\theta}=151$ for $a k$ bins ranging from 0.05 to 0.75 . There are too few samples (21) in the $a k=0.85$ bin to display meaningful results for this last $R e_{\theta}$. As a consequence of the similarity of the surface profiles and velocities, normalized stress results collapse well between the two regimes.

According to Fig. 10, for steepness up to 0.55 the shear is negative everywhere, which is consistent with the present sign convention: the surface normal vector points towards the gas phase and momentum is transferred from the liquid to the gas. At low steepness $(a k=0.05)$, the shear stress along the wave profile is almost constant as expected for a flat surface.

As $a k$ increases the shear stress is modulated similarly to $u_{n}$. The magnitude increases on the upstream facing side $(0 \leq x / \lambda \leq 0.5)$ and decreases on the downstream facing 

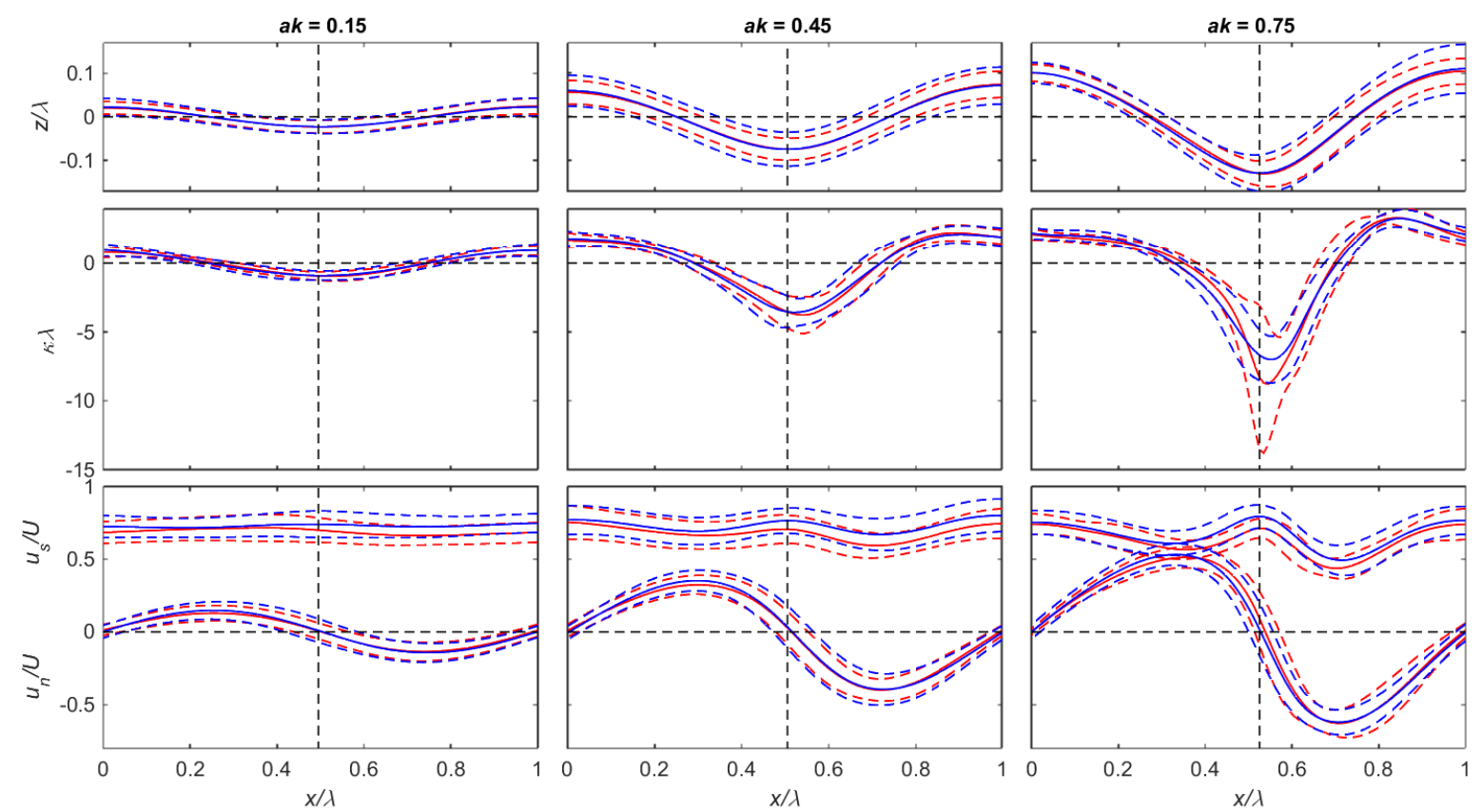

Figure 8: Averaged results (solid line) and RMS (dashed lines) for profile (top), curvature (middle) and surface tangential and normal liquid side velocities (bottom) for the bins $a k=0.15, a k=0.45$, and $a k=0.75$ at $R e_{\theta}=151$ (red lines) and $R e_{\theta}=159$ (blue lines). Vertical dash line marks the trough location.
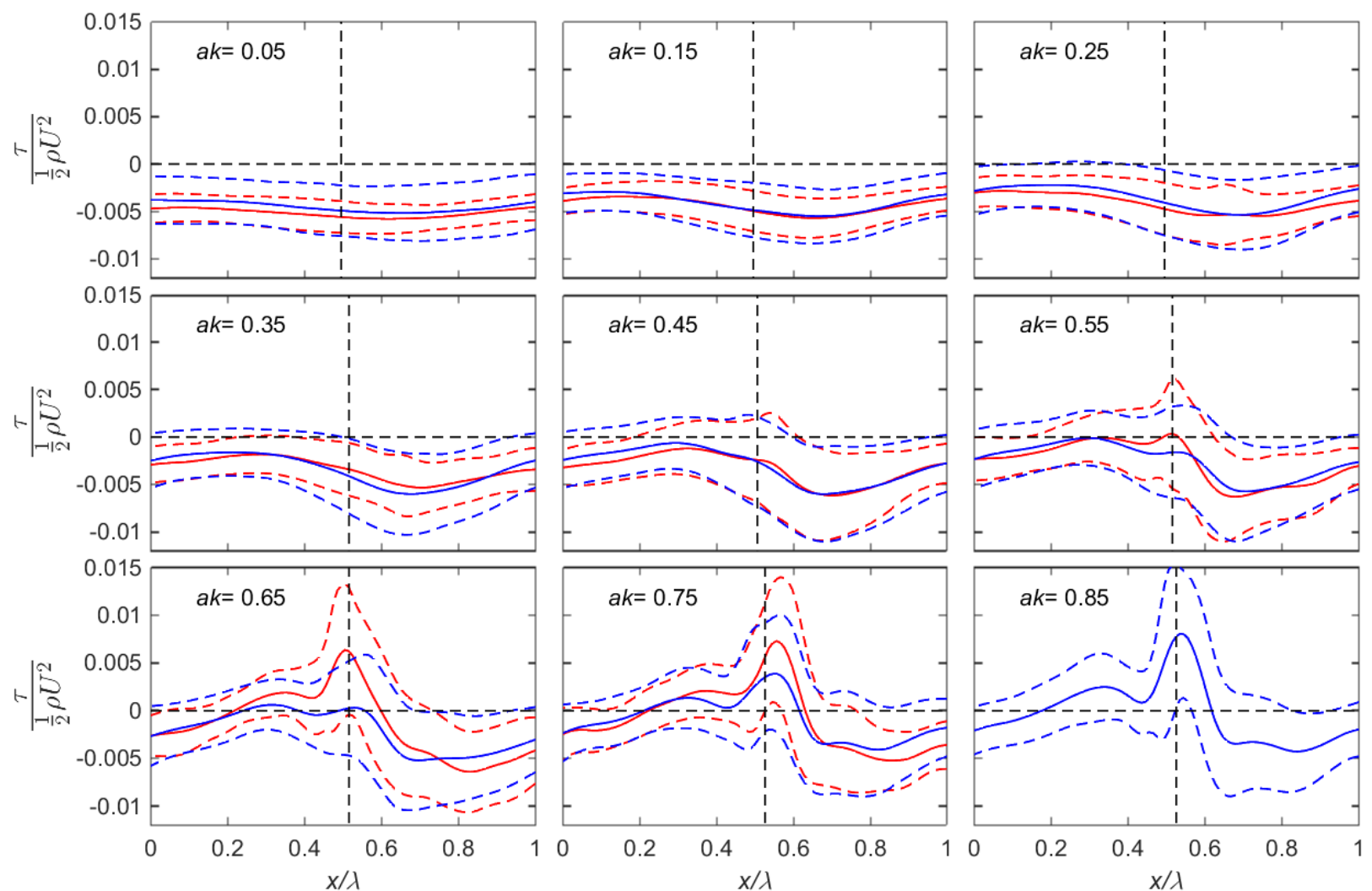

Figure 10: Phase averaged results (solid line) and RMS (dashed lines) for the interfacial shear stress along a wavelength. $R e_{\theta}=151$ is plotted in red for $a k$ bins ranging from 0.05 to 0.75 , and $R e_{\theta}=159$ is plotted in blue for $a k$ bins ranging from 0.05 to 0.85 . Vertical dash line marks the trough location. 

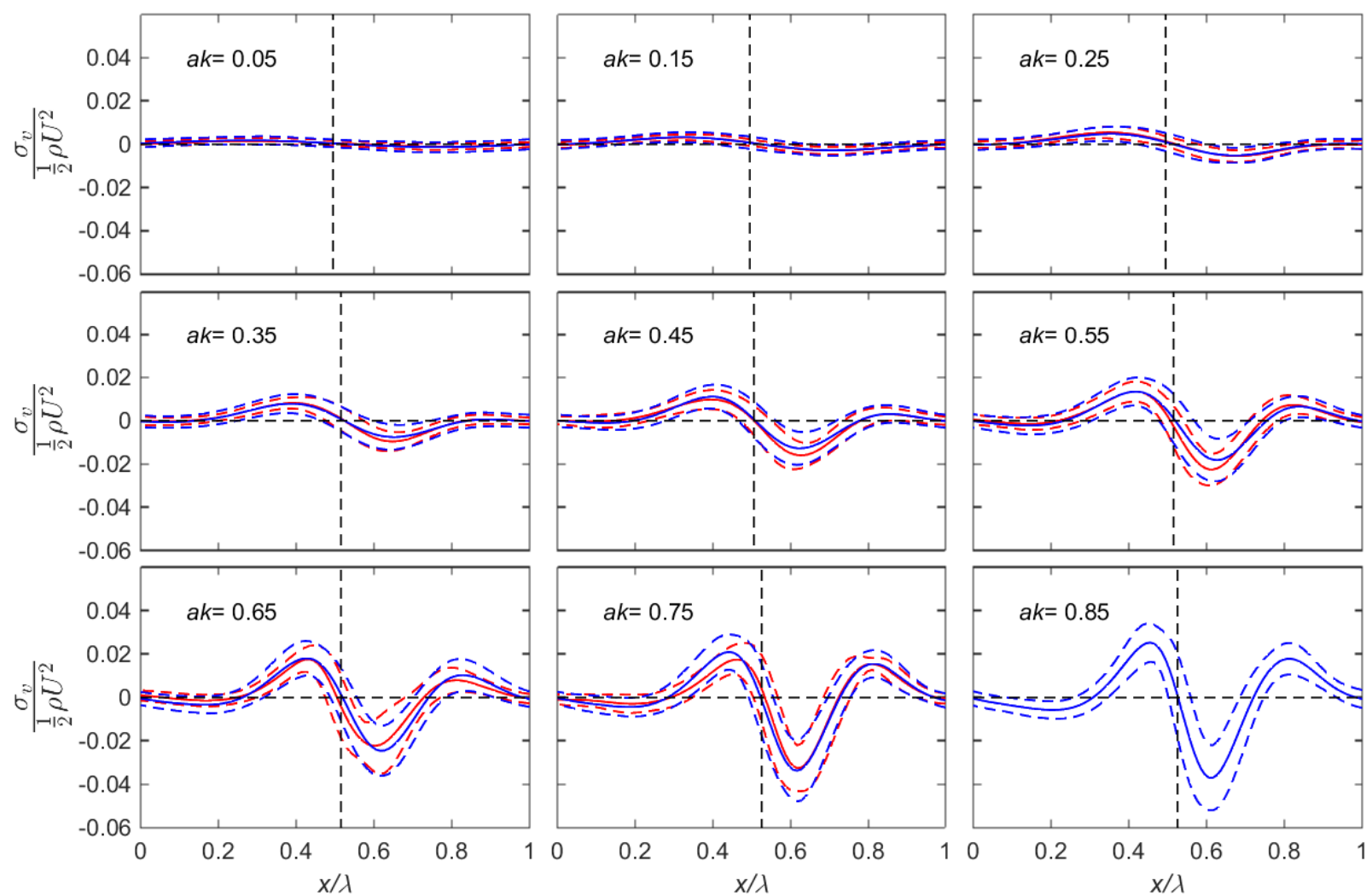

Figure 11: Phase averaged results (solid line) and RMS (dashed lines) for the interfacial normal viscous stress along a wavelength. $R e_{\theta}=151$ is plotted in red for $a k$ bins ranging from 0.05 to 0.75 , and $R e_{\theta}=159$ is plotted in blue for $a k$ bins ranging from 0.05 to 0.85 . Vertical dash line marks the trough location. 

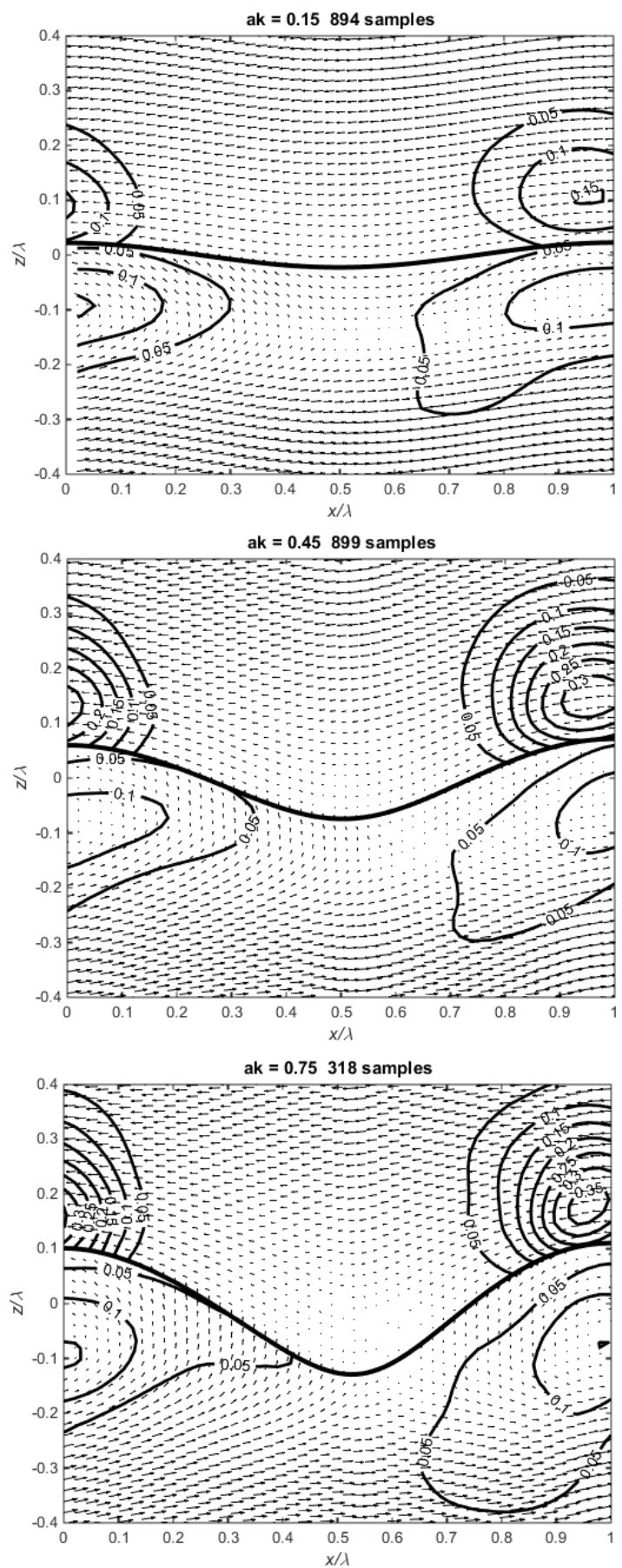

Figure 9: Average vector field for gas and liquid phases for $a k=0.15,0.45$, and 0.75 at $\operatorname{Re}_{\theta}=159$. Contour plot is the normalized swirling strength. Velocity vectors are relative to the wave phase velocity side $(0.5 \leq x / \lambda \leq 1)$. This modulation is symmetrical up to $a k=0.35$.

This trend continues up to $a k=0.55$ although differences become more visible between upstream and downstream regions. In all cases, the shear stress magnitude at the crest decreases by about $50 \%$ as the steepness increases.

At $a k=0.55$, both cases have a shear stress close to zero in the middle of the downstream facing slope $(x / \lambda \approx 0.3)$. At a rigid wall this would indicates a stagnation point. For a moving surface, the Moore Rott Sears (MRS) criterion stipulates that both shear stress and velocity must be zero in unsteady separating flow when viewed in the frame of reference moving with the separation point (Schlichting and Gersten, 2000). Examination of the vector fields reveals that the flow in the gas phase indeed separates in the troughs of the waves, and is responsible for the dramatic change in the distribution of shear stress. This is discussed in more details in Sec. 3.4.

The distribution of normal viscous stress, shown in Fig. 11, is close to zero at low steepness since the surface normal component of velocity remains small. As the steepness increases, a modulation appears, leading to positive stress on the downstream facing slope and negative stress on the upstream facing slope. The normal viscous stress remains negligible at crest and trough. This can be deduced from Eq. 2, by invoking mass conservation in curvilinear coordinates: $\partial u_{n} / \partial n=-\partial u_{s} / \partial s-\kappa u_{n}$. From Fig. $8, u_{n} \approx 0$ and $\partial u_{s} / \partial s \approx 0$ near crest and trough, thus $\sigma_{v}=2 \mu \partial u_{n} / \partial n \approx 0$ there. Above $a k=0.65$, the modulation becomes clearly asymmetrical, with the magnitude downstream of the trough being greater than upstream. Furthermore, a secondary modulation becomes apparent near the crest with negative normal stress in the first quarter of the wave $(0 \leq x / \lambda \leq 0.25)$, and positive stress in the last quarter $(0.75 \leq x / \lambda \leq 1)$. This effect could be related to the increased magnitude of the swirling strength in that region, as shown in Fig. 9. Finally, it should be noted that the amplitude of $\sigma_{v}$ is about 4 times that of $\tau$.

\subsection{Viscous transfer of momentum over a wavelength}

In this section, integration of the stress along the surface yields estimations of the mean momentum transfered via viscous forces over one wave. In Eq. 1 and Eq. 2 the momentum transfer is parallel and perpendicular to the interface, respectively. The latter being curved, horizontal ( $x$-axis) and vertical (z-axis) components of momentum are affected.

Mean momentum transfer is calculated as follow for the shear stress in the horizontal direction: Between two surface data-points, $\tau$ is averaged and multiplied by the local value of the arc length (approximated as a line segment) to yield the local force (per spanwise unit) parallel to the surface. This force is projected in the $x$-direction using the local surface angle $\gamma$ shown in Fig. 1, then integrated along the wave using Simpson's rule (Süli and Mayers, 2003). The result is normalized by the wavelength and becomes equivalent to a mean shear stress over a wavelength, noted $\langle\tau\rangle_{x}$. Contribution of the normal viscous stress, and in the $z$-direction are calculated in a similar manner. 

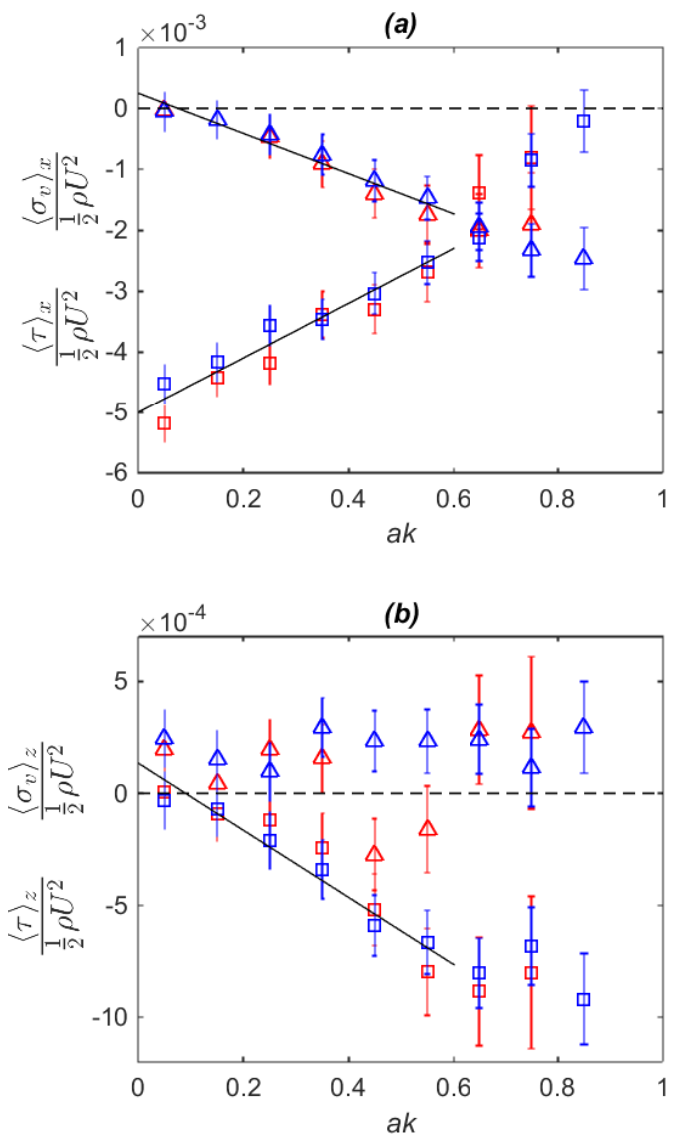

Figure 12: $(a)$ : Mean normal $(\triangle)$ and tangential $(\square)$ viscous stresses in the $x$-direction over a wavelength against wave steepness for $R e_{\theta}=151$ (red) and $\operatorname{Re}_{\theta}=159$ (blue). (b): Same plot for the $z$-direction. Solid lines are linear fits of the data for $0 \leq a k \leq 0.6$, shown in Eq. 3 to 5 . Error bars show the uncertainty at $95 \%$ confidence level.
Results for the dimensionless $x$ - and $z$ - components of mean viscous stresses are displayed in Fig. 12, $(a)$ and $(b)$, respectively for both $R e_{\theta}$. As the steepness increases, $\langle\tau\rangle_{x}$ decreases in magnitude. This trend is also observed for laminar flow over corrugated surface with slip condition (Niavarani and Priezjev, 2009). The decrease is balanced by an increase in magnitude of $\left\langle\sigma_{v}\right\rangle_{x}$, although the overall viscous transfer of horizontal momentum still slightly decreases in magnitude as $a k$ increases.

An attempt at checking the results for flat surface (i.e. negligible normal stress) using a momentum budget on a control volume $(\mathrm{CV})$ containing flow in the gas phase and bounded by the surface was made. However, the momentum transfered through shear stress over one wave is only a small fraction of the incoming momentum. For instance, in the case $R e_{\theta}=151$ and $a k=0.05$ where the viscous shear is the greatest, a CV extending to $z=1 \mathrm{~mm}$ lets in a horizontal momentum flux (per spanwise distance) of $2.2 \times 10^{-3} \mathrm{~kg} / \mathrm{s}^{2}$, while the momentum imparted by the shear stress over a wavelength is only $5.5 \times 10^{-5}$ $\mathrm{kg} / \mathrm{s}^{2} \mathrm{~m}$ or $2.5 \%$. This is on the order of the PIV uncertainty, and thus cannot be used for verification.

The contribution of the shear to the vertical momentum, $\langle\tau\rangle_{z}$, increases in magnitude with the steepness. $\left\langle\sigma_{v}\right\rangle_{z}$ remains close to zero, with a magnitude close to the measurement precision. The net overall trend is a slight increase of momentum transfer with steepness. However, the trend is on the order of the measurement uncertainty, and no definitive conclusions can be drawn here. Nevertheless, this shows that the mean viscous stress in the vertical direction is about an order of magnitude smaller than in the horizontal direction. Such transfer of vertical component could relate to the downward motion of the gas as a consequence of mass conservation during entrainment, as illustrated in Fig. 3, (a).

For $\langle\tau\rangle_{x},\langle\tau\rangle_{z}$, and $\left\langle\sigma_{v}\right\rangle_{x}$, there is a visible transition around $a k=0.6$, consistent with the occurrence of flow separation in the gas phase mentioned before. The mean shear $\langle\tau\rangle_{x}$ is linear with $a k$ before the transition, then tends to zero at a steepness of 0.85 . Equations 3 to 5 fit the linear region $0 \leq a k \leq 0.6$ (bins $a k=0.05$ to 0.55 are used) with a coefficient of determination $R^{2}=0.93,0.96$, and 0.92 , for $\langle\tau\rangle_{x},\left\langle\sigma_{v}\right\rangle_{x}$, and $\langle\tau\rangle_{z}$ respectively. These fits are shown in Fig. 12 as a solid lines. Data for $\left\langle\sigma_{v}\right\rangle_{z}$ are too scattered to produce a good fit.

$$
\begin{aligned}
& \frac{\langle\tau\rangle_{x}}{\frac{1}{2} \rho U^{2}}=+0.0045(a k)-0.0050 \\
& \frac{\left\langle\sigma_{v}\right\rangle_{x}}{\frac{1}{2} \rho U^{2}}=-0.0033(a k)+0.00025 \\
& \frac{\langle\tau\rangle_{z}}{\frac{1}{2} \rho U^{2}}=-0.0015(a k)-0.00014
\end{aligned}
$$

\subsection{Flow separation in the gas phase at high steepness}

Flow separation onset is observed at $a k=0.55$. However, this phenomenon is sporadic up to $a k=0.75$. When averaging vector fields in high steepness bins, separated and attached 


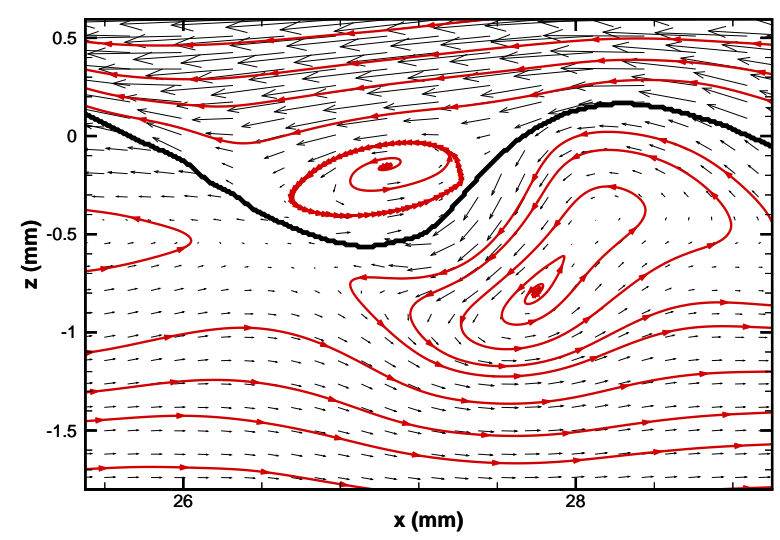

Figure 13: Instantaneous vector field and streamlines in the frame of reference moving with a wave showing flow separation and recirculation zone in the gas phase near the trough. $R e_{\theta}=159$ and $a k=0.85$

flows are combined. This is illustrated in Fig. 9, bottom, where the averaged flow in the trough is not attached nor separated; and in Fig. 10 where the RMS are higher near the troughs at high steepness.

A conditional averaging based on the presence of a recirculation region could be envisioned to deal with unsteady separation, but has not yet been implemented. Furthermore, a larger amount of data would have to be recorded to populate these bins and to obtain meaningful statistics for separation.

An instantaneous vector field where separation occurs at $R e_{\theta}=159$ is presented in Fig. 13. The steepness is 0.85 and a recirculating zone on the gas phase, highlighted by the streamlines, is clearly visible near the trough. The locations of the stagnation point at the surface on the gas side are consistent with the shear stress crossing zero in Fig. 10.

The flow reversal in the separation bubble is responsible for the change of sign of the local shear stress in the trough of high steepness waves as shown in Fig. 10.

\subsection{Contribution of pressure}

The goal of this study is to investigate the effect of wave steepness on the viscous transfer of momentum between liquid and gas. The other contributor to momentum transfer is the pressure term (form drag) of Eq. 2 which arises from nonuniform pressure field or disturbed interface. For instance, Banner (1990) experimentally showed that form drag was dominant over skin friction for a spilling breaker. Pressure was not measured in the present experiment, but its contribution is related to inertia, and thus scales with the Reynolds number. Banner (1990) reported local surface pressure for wind velocity of $2.6 \mathrm{~m} / \mathrm{s}$ to $5.4 \mathrm{~m} / \mathrm{s}$ on $0.4 \mathrm{~m}$ wavelength and 0.3 steepness spilling breaker. The pressure fluctuations over a wave were between $\pm 0.1 \rho U^{2} / 2$, and the wavelength-averaged non-dimensional form drag was around $6 \times 10^{-3} \rho U^{2} / 2$ between adjacent crests. Such magnitude is comparable to that of the viscous measurements of the present study $\left(\langle\tau\rangle_{x}+\left\langle\sigma_{v}\right\rangle_{x}=\right.$
$-4.4 \times 10^{-3} \rho U^{2} / 2$ for $a k=0.3$. Furthermore, due to the smaller scale, the ratio form drag to viscous drag is expected to be lower in the present case compared to the work of Banner. This shows that although form drag is still expected to contribute to momentum transfer, viscous drag likely remains a significant contributor at the present scale below $a k=0.6$.

\section{Conclusions}

Viscous stresses have been measured over a liquid-driven water-air interface populated with $2 \mathrm{D}$ capillary scale disturbances. Using a combination high resolution PIV and PLIF profilometry, the normal and tangential stresses are calculated including contributions of curvature and surface velocity. Data are collected for waves with a steepness ranging from 0 to 1 . Several thousands wave samples are sorted in bins and phaseaveraged to yield statistically meaningful data.

It is found that modulation of the flow by the surface disturbances decreases the shear stress compared to flat surface case while increasing the normal viscous stress. More precisely, viscous stress data show linear trends with respect to the steepness, up to $a k=0.6$. Empirical correlations are proposed based on linear fits of this region.

Above $a k=0.7$, separation occurs in the gas phase, and a recirculation region is observed near the trough. Separation is associated with a sharp drop in the magnitude of shear stress.

Pressure effects (form drag) are not measured here but viscous stresses are compared to literature on surface pressure, and show that unlike spilling breaker for instance, shear stress contribute significantly to the momentum balance in the present flow.

\section{Acknowledgments}

This work was sponsored by the US Office of Naval Research, under the leaderships of Drs. Thomas C. Fu and Ki-Han Kim.

\section{References}

André, M. A., 2014. Measurement of interface dynamics and transfer applied to a free surface shear instability. Ph.D. thesis, The George Washignton University.

André, M. A., Bardet, P. M., 2014. Velocity field, surface profile and curvature resolution of steep and short free-surface waves. Exp. Fluids 55 (4), 1-19.

André, M. A., Bardet, P. M., 2015a. Experimental study of shear layer instability below a free surface. Phys. Fluids 27 (11).

André, M. A., Bardet, P. M., 2015b. Interfacial shear stress measurement using high spatial resolution multiphase PIV. Exp. Fluids 56 (6), 1-18.

Ayati, A., Kolaas, J., Jensen, A., Johnson, G., 2014. A piv investigation of stratified gas-liquid flow in a horizontal pipe. Int. J. Multiph. Flow 61, 129143.

Banner, M. L., 1990. The influence of wave breaking on the surface pressure distribution in windwave interactions. J. Fluid Mech. 211, 463-495.

Belcher, S., Hunt, J., 1993. Turbulent shear flow over slowly moving waves. J. Fluid Mech. 251, 109-148.

Birvalski, M., Tummers, M., Delfos, R., Henkes, R., 2014. Piv measurements of waves and turbulence in stratified horizontal two-phase pipe flow. Int. J. Multiph. Flow 62, 161-173. 
Birvalski, M., Tummers, M., Delfos, R., Henkes, R., 2015. Laminar-turbulent transition and wave-turbulence interaction in stratified horizontal two-phase pipe flow. J. Fluid Mech. 780, 439-456.

Brennen, C., 1970. Cavity surface wave patterns and general appearance. J. Fluid Mech. 44, 33-49.

Crapper, G., August 1957. An exact solution for progressive capillary waves of arbitrary amplitude. J. Fluid Mech. 2 (6), 532-540.

Decarre, S., Sebastien, G., Line, A., Apr. 23 2008. Method for dimensioning industrial installations where a two-phase gas-liquid mixture flows in an intermittent regime. US Patent App. 12/108,026.

Fabre, J., Masbernat, L., Suzanne, C., 1987. Experimental data set no. 7: Stratified flow, part i: Local structure. Multiphase Sci. and Tech. 3 (1-4).

Foucaut, J., Stanislas, M., 2002. Some considerations on the accuracy and frequency response of some derivative filters applied to particle image velocimetry vector fields. Meas. Sci. Technol. 13 (7), 1058.

Ishii, M., Hibiki, T., 2010. Thermo-fluid dynamics of two-phase flow. Springer Science \& Business Media.

Kawaji, M., 1998. Two-phase flow measurements using a photochromic dye activation technique. Nucl. Eng. Design 184 (2), 379-392.

Line, A., Lopez, D., 1997. Two-fluid model of wavy separated two-phase flow. Int. J. Multiph. Flow 23 (6), 1131-1146.

Longuet-Higgins, M., 1992. Capillary rollers and bores. J. Fluid Mech. 240, 659-679.

Lopez, D., 1994. Ecoulements diphasiques stratifiés à faible contenu de liquide. Ph.D. thesis.

Lundgren, T., Koumoutsakos, P., 1999. On the generation of vorticity at a free surface. J. Fluid Mech. 382 (1), 351-366.

Moore, D., 1959. The rise of a gas bubble in a viscous liquid. J. Fluid Mech. $6(01), 113-130$

Niavarani, A., Priezjev, N. V., 2009. The effective slip length and vortex formation in laminar flow over a rough surface. Phys. Fluids 21 (5), 052105.

Peirson, W., 1997. Measurement of surface velocities and shears at a wavy airwater interface using particle image velocimetry. Exp. Fluids 23, 427-437.

Schlichting, H., Gersten, K., 2000. Boundary-layer theory. Springer Science \& Business Media.

Siddiqui, K., Loewen, M. R., 2010. Phase-averaged flow properties beneath microscale breaking waves. Boundary-layer Meteorology 134 (3), 499-523.

Siddiqui, M. K., Loewen, M. R., 2007. Characteristics of the wind drift layer and microscale breaking waves. J. Fluid Mech. 573, 417-456.

Söderberg, L., Alfredsson, P., 1998. Experimental and theoretical stability investigations of plane liquid jets. Eur. J. Mech.-B/Fluids 17 (5), 689-737.

Süli, E., Mayers, D. F., 2003. An introduction to numerical analysis. Cambridge university press.

Taitel, Y., Dukler, A., 1976. A theoretical approach to the lockhart-martinelli correlation for stratified flow. Int. J. Multiph. Flow 2 (5), 591-595.

Veron, F., Saxena, G., Misra, S., 2007. Measurements of the viscous tangential stress in the airflow above wind waves. Geophys. Res. Let. 34 (19).

Whalley, P., 1996. Two-phase flow and heat transfer. Oxford chemistry primers. Oxford University Press.

Zhou, J., Adrian, R., Balachandar, S., Kendall, T., 1999. Mechanisms for generating coherent packets of hairpin vortices in channel flow. J. Fluid Mech. 387, 353-396. 